\section{Fascioliasis: una causa infrecuente de eosinofilia}

\section{Sr. Director:}

La interpretación de los datos analíticos, en ocasiones, se plantea como un reto. Los síntomas y los signos generalmente nos ayudan a valorar acertadamente la relevancia de esas alteraciones. Sin embargo, ante la ausencia de datos clínicos, se puede caer en el error de minusvalorar ciertas alteraciones analíticas y desistir de cualquier esfuerzo diagnóstico.

Presentamos el caso de un paciente, varón de 33 años, previamente sano, remitido por su médico de cabecera por la detección, en una analítica rutinaria, de una marcada eosinofilia (6.660 neutrófilos/ml). No presentaba ningún síntoma, ni signo en el interrogatorio dirigido. No había realizado viajes al extranjero, ni consumido alimentos sin control sanitario. En una nueva analítica, destacaba una notable eosinofilia $(5.680 / \mathrm{ml})$ con discreta elevación de las transaminasas y de la proteína $\mathrm{C}$ reactiva. En el proteinograma llamaba la atención un aumento policlonal de las gammaglobulinas, especialmente de la IgG y de la IgE. Las pruebas alérgicas, las serologías frente a VHA, VHB, VHC y VIH, los parásitos en heces y una serie de autoanticuerpos fueron negativos. En la ecografía se detectó una lesión hepática por lo que se solicitó la determinación de alfa-fetoproteína, que fue normal. Una RNM abdominal mostró varias lesiones hepáticas que por su morfología eran muy sugestivas de fascioliasis hepática. Ante estos hallazgos se pidió serología de diferentes helmintos (fascioliasis, hidatidosis, filariasis, esquistosomiasis, toxocara y teniasis) siendo positiva, a un título de 1:640, los anticuerpos frente a Fasciola hepática. Se administró Triclabendazol $750 \mathrm{mg}$ en dosis única con buena tolerancia y respuesta clínica. En el interrogatorio dirigido no se pudo evidenciar el origen de la infección.

La fascioliasis humana es considerada en la actualidad como una enfermedad tropical reemergente, ya que en los últimos años su incidencia se ha visto aumentada (1). Aún así, en nuestro medio es poco frecuente $(2,3)$. Es una zoonosis producida por un helminto huésped habitual de algunos herbívoros, especialmente las ovejas, cabras y vacas. Su transmisión es fecal-oral a través de quistes depositados en el agua o en ciertas plantas (berros, menta, valeriana, hojas de diente de león, etc.). Una vez en el interior del intestino, los quistes se activan y atraviesan la pared del tubo digestivo, para llegar al hígado penetrando por la cápsula de Glisson. Finalmente, son eliminado por vía biliar.

El período de incubación oscila entre 1 y 3 meses. Se diferencian tres formas clínicas: aguda, crónica y asintomática, que es la más frecuente (2). La forma aguda presenta tres subtipos: la forma típica (80-90\% de los casos de fascioliasis aguda) con fiebre prolongada, dolor abdominal, síndrome constitucional e ictericia; la atípica en la que están implicados mecanismos inmunoalégicos no bien conocidos; y la ectópica, donde el parásito puede ser aislado fuera del hígado.

Para el diagnóstico es necesario, o bien, el aislamiento del parásito en las heces, que sólo es posible en la fase crónica, o la determinación serológica. Esta última es de gran utilidad en los casos de fascioliasis aguda o asintomática ya que presenta una sensibilidad y una especificidad muy elevadas (4-6). En el momento actual el tratamiento de elección es el triclabendazol 5$10 \mathrm{mg} / \mathrm{kg}$ en dosis única $(1,3,7)$. Otras opciones son bitionol 3050 mg/kg/día, 20-30 días o metronidazol 1,5 g/día 13-28 días (8).

Por lo tanto, ante un paciente con eosinofilia de causa no filiada, aún en ausencia de exposición conocida, estaría indicado la realización de serologías frente a helmintos, incluyendo entre ellas la Fasciola hepática.
M. T. Pérez Rodríguez, J. de la Fuente Aguado, S. Pérez Fernández, C. Poderós Baeta ${ }^{1}$, M. Samprón Rodríguez, A. Casteràs Román

Servicios de Medicina Interna y ${ }^{1}$ Hematología. Hospital XeralCíes. Complexo Hospitalario Universitario de Vigo. Vigo, Pontevedra

1. Loja D, Alvizuri J, Vilca M, Avilés R, Sánchez M. Hematoma hepático subcapsular por fasciola. Rev Gastroenterol de Perú 2003; 23: 142-148.

2. Arjona R, Riancho, JA, Aguado JM, Salesa R, González-Macías J. Fascioliasis in developed countries: A review of classic and aberrant forms of the disease. Medicine 1995; 74: 13-23.

3. Carminal L, Fernández C, Quirós JFB, Parra F. Tratamiento de fascioliasis humana con triclabendazol. Rev Clin Esp 1999; 199; 333-335.

4. El-Morshedy H, Farghaly A, Sharaf S, Abou-Basha L, Barakat R. Triclabendazole in the treatment of human fascioliasis: A community based study. East Med Health J 1999; 5: 888-894.

5. Rodríguez R, Carbajal L, Vasquez O, Reynes J, Garcia C, Barrios R, et al. Fascioliasis humana crónica: Informe de un caso tratado con triclabendazol. Acta Pediatr Mex 1999; 20: 23-27

6. Ave J, Alban M, Sagastegui C, Soriano S. Tratamiento de la fascioliasis hepática humana con triclabendazole. Rev Gastroent Perú 1999; 19: 216220

7. Mac Lean JD, Graeme-Cook FM Case 12-2002 - A 50-Year-Old Man with Eosinophilia and Fluctuating Hepatic Lesions. N Eng J Med 2002; 346: 1232-1239.

8. Mansour-Ghanaei F, Shafaghi A, Fallah M. The effect of metronidazole in treating human fascioliasis. Med Sci Monit 2003; 9: 127-130.

\section{Pancreatitis secundaria a paracetamol-codeína}

\section{Sr. Director:}

La pancreatitis aguda (PA) es un problema de salud con una considerable morbi-mortalidad. Estudios epidemiológicos constatan un aumento de las pancreatitis, entre las posibles explicaciones están la mejora de los métodos diagnósticos, el aumento del consumo de alcohol, la realización de procesos pancreatotóxicos y el aumento del consumo de medicamentos $(1,2)$. Presentamos un caso producido por la asociación de paracetamol-codeína.

Varón de 60 años, deportista, sin antecedentes médicos de interés. No bebedor. Sin medicación habitual. Ingresó por presentar de forma brusca un dolor en epigástrico, no irradiado, intenso, que mejoraba con la flexión anterior. No presentaba náuseas ni vómitos. No fiebre ni ictericia. Refería dos horas antes del inicio del dolor, haber tomado un comprimido de paracetamol-codeína como tratamiento sintomático por un cuadro catarral con tos intensa. Resto de la anamnesis sin interés. A la exploración física sólo destacaba un dolor a la palpación en epigastrio sin signos de irritación peritoneal. Exploraciones complementarias: Analítica: amilasemia de 318 (1-100) y amilasuria 1237 (0-460), AST 67 UI/l, ALT $47 \mathrm{UI} / 1$ y bilirrubina total de $1,3 \mathrm{mg} / \mathrm{dl}$. Resto normal. Rx de tórax y abdomen sin hallazgos significativos. Ecografía abdominal: Vesícula normal, sin litiasis. Vía biliar normal. Páncreas de tamaño normal con discreta hipoecogenicidad difusa, compatible con edema. Gastroscopia: esofagitis ${ }^{\circ} \mathrm{I}$ y hernia de hiato. Evolución: Rápida mejoría clínica y completa normalización analítica y ecográfica durante el ingreso. Sin recaída tras 1 año de seguimiento. 